\title{
SIMULAÇÃO DE TÉCNICAS DE ENGENHARIA DE \\ RESERVATÓRIOS: exemplo de utilização de opções reais
}

Richard Saito

Ph.D. em Engenharia Econômica pela Stanford University e Professor do Departamento de Contabilidade, Finanças e Controle da EAESP/FGV.

E-mail: rsaito@fgvsp.br

Denis J. Schiozer

Ph.D. em Engenharia de Petróleo pela Stanford University, Professor do Departamento de Engenharia Mecânica da Unicamp e Chefe do Centro de Estudos de Petróleo da Unicamp.

E-mail:denis@dep.fem.unicamp.br

Guilherme Nogueira de Castro

Engenheiro Elétrico pela Universidade Federal do Rio de Janeiro e Doutorando na EAESP/FGV. E-mail: gcastro@fgvsp.br

\section{RESUMO}

Este trabalho teve como objetivo incluir flexibilidade gerencial (tais como técnicas de recuperação complementar de óleo) na avaliação de reservatórios. Concluímos que essas técnicas podem aumentar o valor dos reservatórios em até $25 \%$ segundo a teoria de opções reais. A principal vantagem da metodologia de teoria de opções em relação à tradicional técnica de fluxo de caixa descontado é levar em conta as questões operacionais da indústria do petróleo. Utilizamos dois modelos clássicos para a precificação de reservatórios de petróleo e aplicamos uma análise de sensibilidade para determinar quais fatores são mais relevantes no seu valor econômico. Como era de se esperar, em ambos os modelos, o tempo de concessão e a taxa de convenience e/ou dividend yield foram os fatores mais importantes.

\section{ABSTRACT}

This paper analyzes the managerial flexibility embedded in oil reserves (such as gas and water injection techniques). We have concluded that these techniques can increase the value of oil reserves by up to $25 \%$ according to the theory of real options. The main advantage of the real options approach over the discounted cash-flow approach is to include the operational issues in the oil and gas industry. We have used two classical models to assess the value of oil reserves. We have also performed sensitivity analysis to confirm that both concession time and convenience and/or dividend yields are the most important factors.

- PALAVRAS-CHAVE: Opções reais, reservas de petróleo, engenharia de reservatórios.

KEY WORDS: Real options, oil and gas reserves, reservoir engineering.$$
\text { ( }
$$ 


\section{INTRODUÇÃO}

A análise do fluxo de caixa descontado (FCD) é a técnica mais conhecida pelos analistas financeiros para determinar o valor econômico de um projeto. Há situações, contudo, em que aspectos estratégicos e operacionais não são considerados na análise do FCD. Por exemplo, suponhamos que uma empresa de petróleo tenha reservas cujo custo de produção seja, no momento da análise, maior que o seu preço de mercado esperado para os próximos anos. Certamente, não há justificativa econômica para iniciarmos a produção de petróleo. Admitamos, no entanto, que, por algum motivo, haja a possibilidade de ocorrer viabilidade econômica devido à volatilidade da conjuntura atual (e.g. falta de abastecimento, aumento de impostos de importação, etc.) e que, conseqüentemente, seja interessante termos a opção de explorar a reserva. As possibilidades de interromper, de aumentar e/ou diminuir e de iniciar a produção representam claramente opções. Essas opções têm um valor econômico aos seus administradores. Em particular, a área de exploração, desenvolvimento e produção de petróleo e gás representa uma oportunidade de aplicarmos a teoria de opções reais ${ }^{1}$ para sua avaliação.

$\mathrm{Na}$ indústria de petróleo e gás, é comum os administradores esperarem um ano ou mais antes de decidir pela exploração e/ou desenvolvimento de uma reserva. Algumas razões para a espera podem incluir: a) mais certeza sobre o mercado internacional de petróleo; b) melhores custos de capital para o financiamento do projeto; c) desenvolvimento do projeto em dois estágios para avaliar o potencial da reserva; d) criação de novas tecnologias de exploração, desenvolvimento e produção; e e) negociação de melhores parceiros tecnológicos e comerciais. Para esses casos, o valor da opção de esperar pode ser alto. Uma implicação interessante é que um projeto com valor presente líquido negativo hoje pode tornar-se viável amanhã e justamente essa diferença é representada pelo valor da opção de flexibilidade dada aos administradores.

A literatura de opções reais aplicadas a problemas de orçamento de capital é extensa. Particularmente, salientamos os trabalhos de Brennan e Schwartz (1985) e de Paddock, Siegel e Smith (1988) para avaliação de reservas de recursos naturais. A metodologia utilizada por ambos é a aplicação da teoria de opções e do modelo de equilíbrio em mercadorias (commodities) para a avaliação de reservas naturais. Neste estudo, implementamos e adaptamos os dois trabalhos para fazer a comparação com as principais práticas da indústria. Essas práticas incluem: a) o parâmetro de US\$ 3 por barril usado pelos bancos de investimentos ${ }^{2}$ para a precificação de reservas a desenvolver e b) o valor de um terço do preço de mercado ${ }^{3}$ para precificar as reservas segundo especialistas do mercado.

Este artigo é oportuno por analisar o valor econômico dada a concessão pelo Governo Federal à iniciativa privada da exploração, do desenvolvimento e da
É oportuno analisar o valor econômico dada a concessão pelo Governo Federal à iniciativa privada da exploração, do desenvolvimento e da produção de reservas de petróleo e gás. produção de reservas de petróleo e gás. Essa concessão pode incluir não somente a exploração de uma reserva de petróleo mas também o seu desenvolvimento e a sua produção. A oportunidade de concessão ao setor privado é consequiência das limitações de investimento da Petrobras, bem como da desregulamentação do setor de petróleo. Para os próximos cinco anos, a Petrobras pretende firmar parcerias para desenvolver projetos no valor de US\$ 75 bilhões, além de empreendimentos consorciados na indústria de petróleo e gás. De acordo com o governo, a produção brasileira deve passar dos atuais 940 milhões de barris por dia (bpd) para mais de 1,5 bilhão bpd em 2005, como resultado desse programa de investimento.

Em contrapartida à concessão à iniciativa privada, o Governo Federal receberá royalties, além do aluguel da área concessionada. $\mathrm{O}$ valor do royalty a ser pago pelo concessionário ao governo representa um dos principais tópicos de interesse do regulador. A regulamentação da exploração, do desenvolvimento e da produção de reservas de petróleo cabe à Agência Nacional de Petróleo (ANP) ${ }^{4}$, autarquia vinculada ao Ministério das Minas e Energia.

Este estudo tem como objetivo apresentar, implementar, comparar e criticar as metodologias utilizadas para a precificação de reservas de petróleo. A nossa contribuição consiste em avaliar as vantagens e as desvantagens de cada modelo de acordo com as incertezas inerentes ao processo de exploração, de desenvol- 
vimento e de produção. Para cada uma dessas fases, temos variáveis mais críticas quanto à sua incerteza e, para tanto, o modelo apropriado ou mais recomendado deve ser levado em consideração. Isso deve ser feito conciliando-se os aspectos tecnológicos das reservas brasileiras e aliando-os às práticas de engenharia financeira e de engenharia de simulação de reservatórios. Os resultados mostram-nos a sensibilidade do valor das reservas quanto à tecnologia, à volatilidade dos preços do petróleo e ao modelo de opções utilizado.

Os resultados mostram-nos a sensibilidade do valor das reservas quanto à tecnologia, à volatilidade dos preços do petróleo e ao modelo de opções utilizado.

Organizamos este artigo como se segue. Na segunda seção, revemos as características de uma reserva de petróleo e descrevemos sua precificação com a utilização da teoria de opções. A terceira seção descreve os modelos de Brennan e Schwartz (1985) e de Paddock, Siegel e Smith (1988) e os implementa em código de computador sob três condições de tecnologia de exploração (isto é, sem injeção, com injeção de água e com injeção de gás). Os resultados são apresentados e analisados na quarta seção. Comentários finais e sugestões para futuras pesquisas são objetos da quinta seção.

\section{RESERVAS DE PETRÓLEO COMO OPÇÕES REAIS}

Quando nos referimos a opções, geralmente associamos isso à opção de compra ou de venda de um ativo financeiro. No caso de opções reais, os ativosobjetos correspondentes são ativos reais. Por exemplo, imagine que um empreendedor tenha um projeto de construir um hotel de 40 suítes e, para tanto, ele tenha a opção de fazê-lo em duas fases: na primeira fase, ele pode construir a infra-estrutura e as primeiras 20 suítes e, em uma segunda fase, dependendo do crescimento da demanda, ele pode optar por completar o projeto original do hotel.

O exemplo anterior representa uma opção real dada ao empreendedor de ter flexibilidade gerencial, respondendo de acordo com as condições de mercado. Os tipos de opções reais podem ser classificados como ${ }^{5}$ : opção de crescer, opção de expandir, opção de esperar, opção de contratar escala adicional e opção de abandonar.

No caso de indústrias intensivas em capital, como no caso de petróleo e gás, as opções de abandono representam uma alternativa no caso de, por exemplo, a margem de contribuição diminuir consideravelmente. Também é natural encontrarmos a opção de esperar o desenvolvimento e a produção de uma reserva de petróleo, já que o seu início é irreversível. A opção de comprar, contrair ou aumentar a escala representa uma alternativa de atender à demanda sem incorrer em custos fixos desnecessários. A opção de mudar o mix de produção, seus insumos ou ainda os processos de produção pode ser encontrada no uso da terra para diferentes culturas, de trabalhadores com habilidades diversas e de planta de co-geração que pode tanto usar carvão como gás natural.

Especificamente para o caso de reservas de petróleo, temos três fases: exploração, desenvolvimento e produção. A fase de exploração envolve atividades de perfuração e estudos sísmicos para obter informações sobre as quantidades de reservas de hidrocarboneto, bem como os seus custos (e as tecnologias mais adequadas) para extraí-las. A exploração de uma potencial reserva tem como objetivos: a) determinar o tamanho do reservatório em termos; b) averiguar a qualidade do meio de transmissão do hidrocarboneto (e.g. viscosidade do óleo e porosidade da rocha); e c) identificar a qualidade do óleo (e.g. densidade e mix de hidrocarbonetos). Após a fase de exploração, o concessionário poderá mapear o reservatório de forma a estabelecer os poços e o uso de tecnologia de modo a extrair petróleo e gás em maior quantidade ao menor custo.

O segundo estágio refere-se ao desenvolvimento da reserva, ou seja, uma vez identificado o potencial de hidrocarbonetos da reserva e a tecnologia para desenvolvê-lo, o concessionário tem a opção de realizar as perfurações e instalar as plataformas de produção para iniciar a extração de petróleo e gás. Durante essa fase, a engenharia de petróleo pela simulação de reservatórios é extensivamente utilizada para determinar justamente o plano de desenvolvimento do reservatório.

Finalmente, durante o estágio de produção, o concessionário pode exercer a opção de extrair os hidrocarbonetos. Para exercer essa opção, ele deve levar em consideração alguns parâmetros, tais como a qualidade do petróleo, as expectativas futuras de extração e os seus custos, a política de impostos e royalties ao governo e os preços dos hidrocarbonetos. 
Tais parâmetros relacionados ao mercado podem ser determinados com razoável tolerância. Contudo, aspectos de operação e tecnológicos são mais difíceis de determinar para reservas em geral.

A determinação do potencial de um reservatório de petróleo é extremamente difícil, dada a sua complexidade. As características da rocha, a temperatura, a viscosidade dos fluidos, a pressão e até mesmo a forma do reservatório são fatores que influenciam profundamente o comportamento dos fluidos e indicam a viabilidade ou a inviabilidade de seu desenvolvimento e exploração. Em muitos casos, é decidido que reservatórios não são economicamente viáveis, apesar de possuírem quantidades bastante significativas de óleo e gás.

Com o recente avanço das ferramentas computacionais, podemos simular um reservatório por meio da modelagem de cada elemento infinitesimal, como no caso do uso de métodos finitos para cálculo estrutural. Tipicamente, um modelo simula como as ações de uma sonda combinada com outras sondas que podem injetar gás ou água fazem fluir o óleo (com maior pressão) pela sonda até a superfície. Conforme a continuidade da produção, a pressão inferior, que era maior, dilui-se e necessita-se de alternativas para a extração dos hidrocarbonetos. O material que chega à superfície é óleo cru quente, algumas vezes acompanhado de gás natural. O óleo cru é uma mistura de petróleo líquido e gases em várias combinações. Cada um dos componentes possui algum tipo de valor, mas somente depois de isolados por um processo de refino.

Justamente para melhorar o rendimento da extração de óleo, utilizam-se técnicas que possibilitam um aumento de pressão para dar continuidade do fluxo de petróleo à superfície. As técnicas incluem injeção de água, injeção de gás e produtos químicos que funcionam como detergentes (que fazem com que o óleo incrustado nas rochas se separe delas), injeção de vapor e aumento de temperatura.

Como podemos ver na Figura 1, a água é o fluido localizado nas maiores profundidades, estando abaixo dos demais. O método da injeção de água consiste na colocação de mais água, por meio de poços localizados estrategicamente, no fundo do reservatório para que aumente a pressão existente. Devido a esse procedimento, a pressão abaixo do óleo aumenta e o empurra para cima, fazendo com que este flua para os poços produtores. A injeção de gás é o outro método bastante utilizado para a melhor recuperação de óleo existente nos reservatórios. Por meio de poços injetores de gás, nos quais existem compressores para empurrar a cobertura de gás para baixo, aumenta-se a pressão existente sobre o óleo. Como a pressão existente abaixo do óleo é maior do que nos poços produtores, quando o gás empurra o óleo para baixo, o óleo entra nas estações produtoras e flui para a superfície. ${ }^{6}$

A idéia básica é que a pressão existente no reservatório direcione o óleo à superfície. Dada a grande complexidade das características dos fluidos em função das condições locais de pressão e temperatura, utilizamos técnicas de simulação de reservatórios para obter a maior extração possível do óleo a um baixo custo.

A principal contribuição deste estudo é poder integrar técnicas recentes de engenharia de simulação de reservatórios com técnicas de precificação de opções reais. Particularmente, isso se mostra conveniente na medida em que, nos últimos anos, as técnicas de recuperação de óleo e gás têm evoluído para o uso combinado de injeção de gás e de água.

\section{Figura 1 - Técnicas de recuperação de óleo: injeção de água e de gás}

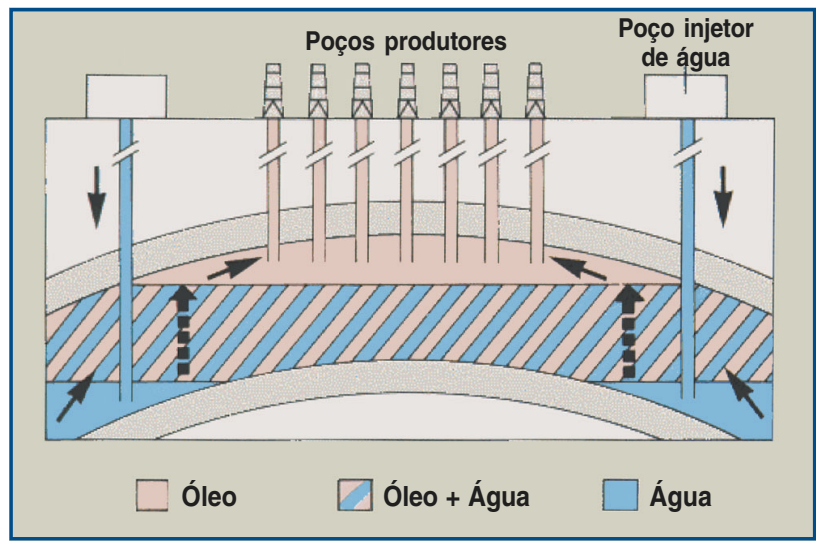

Injeção de água

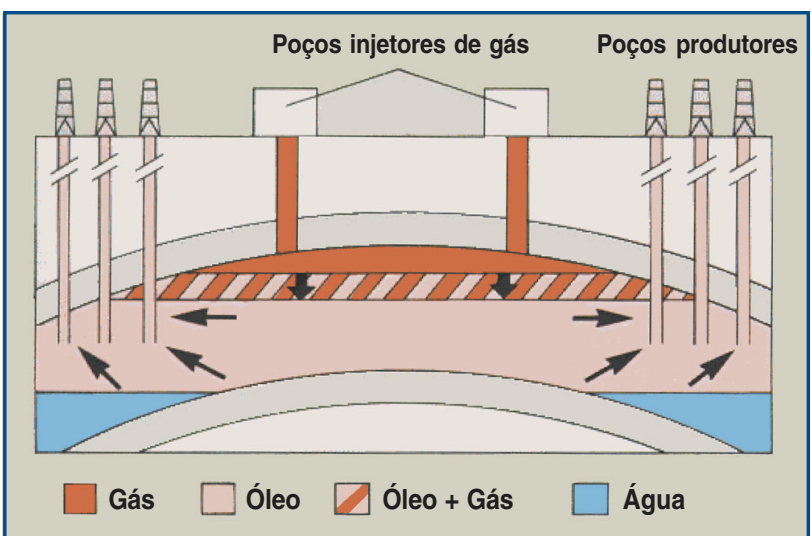

Injeção de gás 


\section{AVALIAÇÃO DE RESERVAS PETROLÍFERAS}

Alternativamente à abordagem tradicional de fluxo de caixa descontado, utilizamos a teoria de opções reais para determinarmos o valor de contratos de concessão de exploração de reservas de petróleo. Escolhemos dois modelos para avaliar os reservatórios: o modelo de Paddock, Siegel e Smith (1988) e o modelo de Brennan e Schwartz (1985), revisados abaixo. Em seguida, ressaltamos as principais diferenças entre os dois modelos e, finalmente, apresentamos os parâmetros utilizados no uso dos modelos.

Há dois métodos para determinar o valor de opções cujos ativos-objetos seguem um processo estocástico browniano. O primeiro, utilizado por Brennan e Schwartz (1985), replica uma carteira cujo risco é nulo e é descontado a taxa livre de risco. Para anularmos a

cificado um problema de avaliação em detalhe suficiente para examinar os problemas teóricos e práticos envolvendo a extensão da teoria de avaliação de opções financeiras para casos reais. Nesse momento, são descritas as diferenças entre as opções financeiras e as opções reais e é mostrado como os obstáculos que surgem dessa analogia podem ser superados.

Incluindo as modificações necessárias na equação de Black-Scholes para que esta calcule o valor de uma opção americana que paga dividendos, chegamos à seguinte equação:

$$
\frac{\partial X}{\partial t}=r X-(r-\delta) S \frac{\partial X}{\partial S}-\frac{1}{2} \sigma_{S}^{2} S^{2} \frac{\partial^{2} X}{\partial S^{2}}
$$

em que: $r$ é a taxa livre de risco, $S$ é o valor do reservatório, $X$ é o custo de explorá-lo (ou desenvolvê-lo, dependendo da fase, e representa o nosso preço de exercício), $\sigma_{S}^{2}$ é a variância do valor do reservatório e $\delta$ é o correspondente pagamento de dividendos (representado pelo lucro operacional da reserva). O exemplo a seguir mostra como podemos utilizar esse modelo.

Consideremos a seguinte versão simplificada para avaliar uma propriedade offshore com reservas estimadas em 50 milhões de barris de petróleo, em que o valor presente de desenvolvimento é US\$ 12 por barril e o tempo para desenvolvê-la é de dois anos. A empresa tem a concessão de explorar essa reserva nos próximos 20 anos, e o valor marginal por barril de petróleo é US\$ 12 por barril (isto é, preço por barril = custo mar-

estocasticidade da carteira, determinamos a função diferencial do derivativo correspondente por meio do Lema de Itô. O segundo método desenvolve um modelo de equilíbrio para o ativo-objeto e este é integrado ao modelo de opções, conforme desenvolvido por Paddock, Siegel e Smith (1988), cuja derivação é baseada no trabalho de McDonald e Siegel (1983).

\section{O modelo de Paddock, Siegel e Smith}

O modelo desenvolvido por Paddock, Siegel e Smith (1988) precifica concessões de reservas de petróleo offshore ou reservas já desenvolvidas. O desenvolvimento do modelo segue os seguintes passos: a) demonstrase como integrar um modelo explícito de equilíbrio de mercado para o ativo real - no caso, reservas de petróleo desenvolvidas - utilizando a teoria de opções para derivar o seu valor correspondente (esse desenvolvimento é importante para que a avaliação do valor da opção seja feita dentro de um cenário livre de risco); e b) usando-se um contrato de concessão como exemplo, é espe- ginal por barril). Uma vez desenvolvida, a produção anual corresponde a $5 \%$ do valor das reservas. A taxa livre de risco é $8 \%$ e o desvio-padrão de $\ln$ (preço do petróleo) é $3 \%$.

Uma simples forma de calcular o valor da reserva é utilizar a equação de Black-Scholes para estimar o valor dessa opção real:

a) valor corrente do ativo, $V$, ou seja, valor da reserva desenvolvida trazida a valor presente: US\$ 12 × 50 $/(1,05)^{2}=$ US\$ 544,22 milhões;

b) preço de exercício, $X$, valor presente do custo de desenvolvimento: US\$ 12 x $50=$ US\$ 600 milhões;

c) tempo em que expira a opção: 20 anos;

d) desvio-padrão no valor do ativo-objeto: $3 \%$;

e) taxa livre de risco: $8 \%$;

f) dividend yield $=$ valor da produção anual/valor da reserva: $5 \%$.

A partir dessas informações, calculamos os parâmetros $d_{1}=1.0359$ e $d_{2}=0.2613$. Isso implica um valor da opção igual a US\$ 97 milhões ${ }^{7}$, ou seja, apesar 
do alto custo de desenvolvimento, o reservatório tem um valor econômico, pois há possibilidade de os preços aumentarem no futuro.

\section{O modelo de Brennan e Schwartz}

O modelo de Brennan e Schwartz (1985) é desenvolvido para recursos naturais, e não especificamente para a indústria de petróleo como o anterior. $\mathrm{O}$ modelo baseia-se em encontrar um portfólio que possua a propriedade de replicar os fluxos de caixa a serem avaliados. O valor presente dos fluxos de caixa é, portanto, igual ao valor corrente do portfólio replicado. As premissas limitadoras do modelo, apontadas pelos autores, são: ${ }^{8}$ a) os recursos explorados são homogêneos ao longo do tempo; b) a quantidade de recursos existentes na jazida é conhecida, ou seja, em casos que envolvem a etapa de exploração de petróleo, este modelo não é adequado, sendo útil apenas para o caso de reservatórios já desenvolvidos; c) os custos são conhecidos; e d) as taxas de juros são não-estocásticas. A equação de Brennan e Schwartz para a avaliação de opções é apresentada abaixo:

$$
\frac{\partial X}{\partial t}=r X-\left(r-\frac{C}{S}\right) S \frac{\partial X}{\partial S}-\frac{1}{2} \sigma^{2} S^{2} \frac{\partial^{2} X}{\partial S^{2}}
$$

em que: $C$ é a convenience yield ${ }^{9}$ para o mercado de petróleo. Os outros parâmetros são os mesmos da equação do modelo de Paddock, Siegel e Smith (1988).

\section{Diferenças entre os modelos de opções}

O modelo de Paddock, Siegel e Smith (1988) desenvolve-se baseado numa análise de equilíbrio de mercado, enquanto o modelo de Brennan e Schwartz (1985) usa um portfólio autofinanciado para o seu desenvolvimento. Uma diferença importante entre os modelos é a premissa quanto à produção de óleo durante a vida útil do reservatório. O modelo de Paddock, Siegel e Smith (1988) considera que o decaimento da produção é exponencial, enquanto o modelo de Brennan e Schwartz (1985) parte da premissa de que a taxa de produção é constante. Em reservatórios reais, a taxa de produção cai exponencialmente, mas a produção, em quase todos os casos, é dimensionada para que os equipamentos e as tubulações tenham a sua utilização maximizada no tempo.

Uma outra diferença importante é o parâmetro utilizado como o retorno devido à utilização do reserva- tório como fonte de rendimentos. No modelo de Paddock, Siegel e Smith, é utilizada uma variável equivalente ao dividendo de uma ação, enquanto, no modelo de Brennan e Schwartz, usa-se a convenience yield. reservatório hipotético possui um poço produtor e um poço injetor. $\mathrm{O}$ modelo numérico de simulação do campo é um modelo de diferenças initas, sendo o reservatório dividido em blocos, e o modelo simula o fluxo do petróleo ao longo do tempo.

\section{Cálculo dos parâmetros}

Os principais parâmetros para o uso do modelo são:

Valor da reserva desenvolvida: seguindo o estudo de Gruy, Garb e Wood (1982), há uma prática de mercado em que o valor da reserva desenvolvida equivale a um terço do valor do preço do óleo cru no mercado. Portanto, como o preço do óleo cru utilizado foi de US\$ 16 por barril, o valor de uma reserva desenvolvida é aproximadamente US\$ 5,33 por barril.

Desvio-padrão do valor da reserva: utilizamos como proxy ao desvio-padrão do valor das reservas o desvio-padrão do preço do óleo cru, pela sua liquidez e pela confiabilidade e transparência na divulgação dos seus dados. Os valores trimestrais dos preços à vista do óleo cru possuem um desvio-padrão, $\sigma$, igual a $3,96 \%$ ao ano para o período de 1985 a 1998.

Custos de exploração e desenvolvimento: os custos de exploração e desenvolvimento utilizados foram os dados de uma pesquisa realizada em 1998 no Canadá pela empresa Peters \& Co. Limited, especializada na indústria de petróleo. Utilizando uma amostra de $74 \mathrm{em}$ presas de petróleo, estimamos o valor de US\$ 5,54 por barril em relação aos custos de exploração e desenvolvimento. Esses valores já foram trazidos a valor presente, considerando o período relativo às operações. Consideramos que o tempo de desenvolvimento para um reservatório offshore seja de três anos em média.

Tempo de concessão: o tempo de concessão foi de 10 anos, correspondente às simulações para o reservatório hipotético. 
Taxa livre de risco: a taxa livre de risco utilizada foi a média dos retornos dos títulos do tesouro americano para um período de 10 anos referentes ao mês de setembro de 1998 . O valor utilizado foi igual a $4,27 \%$ ao ano.

Receita líquida menos depleção: a receita líquida utilizada menos a depleção ${ }^{10}$ foi encontrada utilizandose quatro premissas básicas: a) o valor de uma reserva desenvolvida por barril é igual a um terço do preço de mercado do óleo cru; b) os custos operacionais da Petrobras são de US\$ 6,40 por barril (também foi feita uma análise utilizando-se o valor de US $\$ 4,80$ por barril, já que a maioria das empresas possui custos operacionais dentro dessa faixa de valores); c) a depreciação representa $20 \%$ do preço de mercado do óleo cru; e d) a taxa de produção anual é considerada de $10 \%$ ao ano, dado que o período estudado é de 10 anos. O valor calculado é de $2,47 \%$ ao ano (equivalente ao conceito de dividend yield).

Convenience yield: o valor utilizado para a convenience yield foi de $1 \%$, seguindo o trabalho de Brennan e Schwartz (1985).

\section{APLICAÇÃO A UM RESERVATÓRIO HIPOTÉTICO}

Para mostrar um exemplo de como o modelo pode ser aplicado na prática, utilizamos um reservatório hipotético. Organizamos esse exemplo de aplicação como se segue. Primeiramente, é apresentado o modelo do reservatório. Os valores dos parâmetros são aqueles já descritos anteriormente. Os resultados são apresentados e, finalmente, é demonstrada a sensibilidade dos valores dos reservatórios às variações dos parâmetros.

\section{Descrição do reservatório}

O reservatório hipotético possui um poço produtor e um poço injetor (de gás ou água). O modelo numérico de simulação do campo é um modelo de diferenças finitas, sendo o reservatório dividido em blocos (no caso do exemplo, em 300 blocos), e o modelo simula o fluxo do petróleo ao longo do tempo.

Para a simulação, consideramos um poço produtor - poço 1 - no bloco central do reservatório, como se faz na prática, e foram analisados três casos separados. No primeiro caso - Caso0 -, apenas as forças naturais existentes num reservatório, já discutidas, foram utilizadas para a produção de óleo. No segundo caso - CasoGas -, além das forças naturais, houve injeção de gás por um segundo poço - poço 2 - para um aumento no fator de recuperação do reservatório. E, por fim, no terceiro caso - CasoAgua -, houve injeção de água pelo poço 3 . O esquema é apresentado na Figura 2.

\section{Figura 2 - Reservatório hipotético}

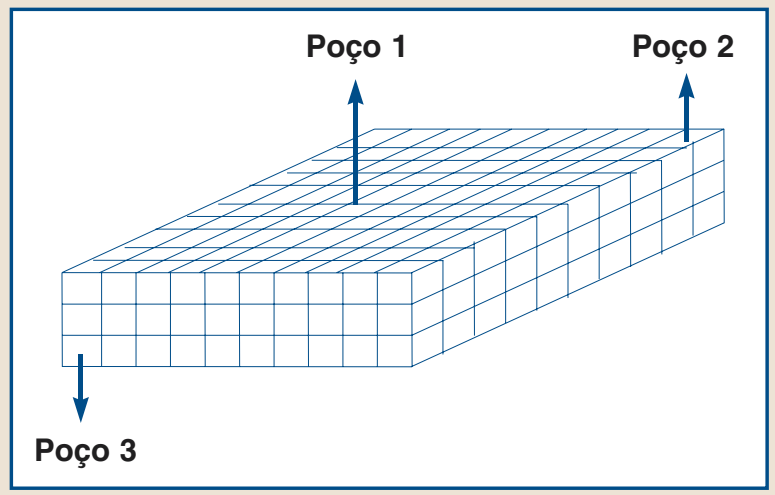

\section{Figura 3 - Taxas anuais de produção sob três técnicas}

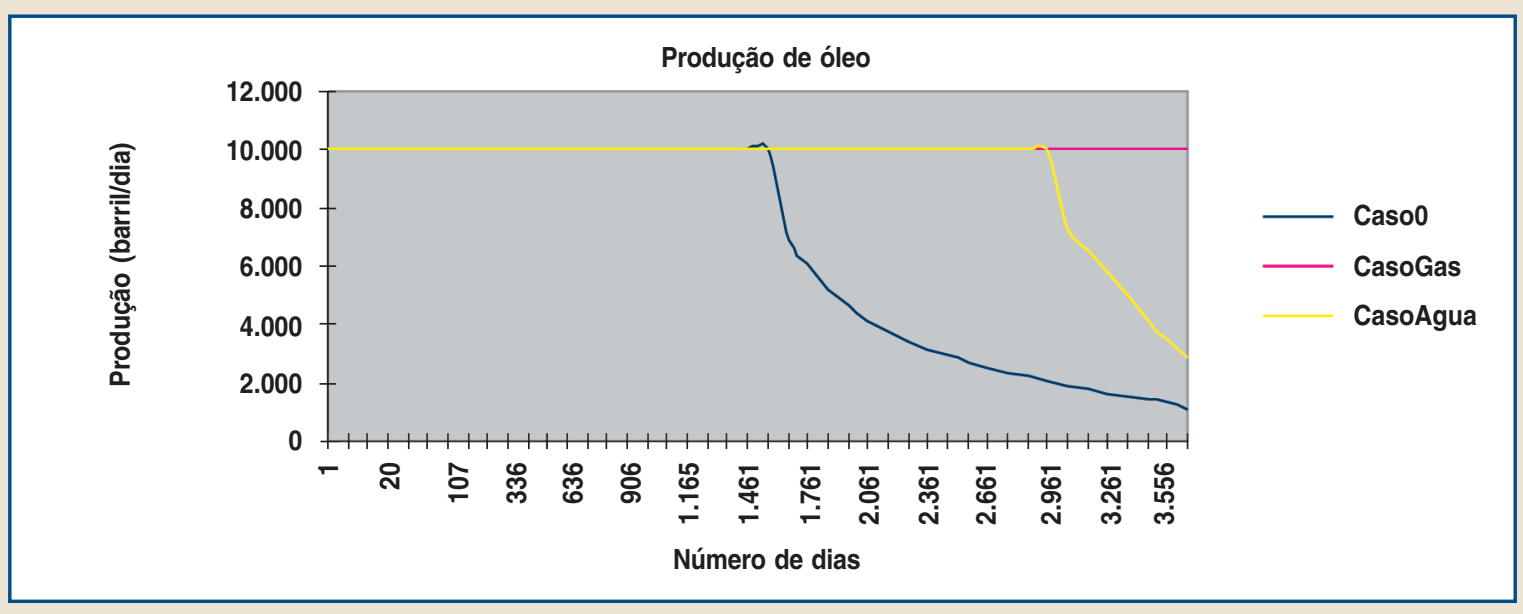


Nos três casos, foi imposta a condição de extração de 10 mil barris diários do poço produtor (poço 1). Além disso, foi imposta uma condição de parada após 10 anos. Dadas essas condições, simulamos o comportamento do reservatório para obtermos as taxas anuais de produção para os três casos, mostradas na Figura 3.

A produção total do reservatório ao longo de 10 anos para cada caso é de: a) Caso0: 25,7 milhões de barris; b) CasoAgua: 35,6 milhões de barris; e c) CasoGas: 36,5 milhões de barris.

\section{Resultados}

Os resultados encontrados estão nas Tabelas 1 e 2 . As equações diferenciais parciais, por não possuírem solução analítica, foram resolvidas numericamente, utilizando o método das diferenças finitas explícitas ${ }^{11}$.

Para utilizar as Tabelas 1 e 2, procede-se da seguinte forma:

1. Calcula-se o valor presente de uma reserva desenvolvida. Como comentado anteriormente, esse passo já foi feito, chegando-se ao valor de $S=$ US $\$ 5,54$ por barril.

2. Calcula-se o custo de seu desenvolvimento e, conseqüientemente, a razão $S / X=5,33 / 5,54=0,96$.
3. Calcula-se o valor da reserva a ser desenvolvida das Tabelas 1 e 2 e do parâmetro $S / X=0,96$.

Temos, para o Caso0: a) segundo a Tabela 1 (modelo de Paddock, Siegel e Smith): valor do reservatório será igual a 0,16687 x 5,54 x 25,7 milhões de barris $=$ US $\$ 23,8$ milhões, e b) segundo a Tabela 2 (modelo de Brennan e Schwartz): o valor do reservatório será igual a 0,17714 x 5,54 x 25,7 milhões, que resulta em um valor econômico igual a US\$ 25,2 milhões.

A avaliação anterior considerou o Caso0. Mas quais seriam os valores dos reservatórios com técnicas de injeção de gás e de água? Para compararmos os valores segundo esses três critérios de extração, utilizamos as seguintes premissas: a) o custo de um poço injeto (a água ou a gás) foi fixado em US\$ 7,5 milhões; b) o custo de desenvolvimento é assumidamente igual a US\$ 5,54 por barril - mais o custo do(s) poço(s) injetor(es) para o CasoAgua e o CasoGas ${ }^{12}$; c) os custos operacionais no CasoAgua e no CasoGas serão maiores, sendo que o custo de injeção de água é de US\$ 0,50 por barril e o de injeção de gás é de US\$ 0,10 por barril ${ }^{13}$.

A partir das considerações do trade-off entre custo adicional de desenvolvimento e produção vis-à-vis a

Tabela 1 - Valor da opção segundo o modelo de Paddock, Siegel e Smith

\begin{tabular}{|c|c|c|c|c|c|c|c|c|c|c|c|c|}
\hline \multicolumn{13}{|c|}{ CÁLCULO DO VALOR DA OPÇÃO PELO MODELO DE PADDOCK, SIEGEL E SMITH } \\
\hline \multicolumn{13}{|c|}{$\sigma=3,96 \%, r=4,27 \%$ e $O C=6,4$} \\
\hline$s / X$ & $t=0$ & $t=3$ & $t=6$ & $t=9$ & $t=10$ & $t=12$ & $t=15$ & $t=18$ & $t=21$ & $t=24$ & $t=27$ & $t=30$ \\
\hline \multicolumn{13}{|l|}{ Out-of-the-money } \\
\hline 0,80 & 0,00000 & 0,00000 & 0,00000 & 0,00908 & 0,02242 & 0,03034 & 0,06245 & 0,10179 & 0,14438 & 0,18695 & 0,22738 & 0,26467 \\
\hline 0,85 & 0,00000 & 0,00000 & 0,01172 & 0,04961 & 0,05051 & 0,09208 & 0,13890 & 0,18576 & 0,23018 & 0,27118 & 0,30876 & 0,34335 \\
\hline 0,90 & 0,00000 & 0,00000 & 0,02366 & 0,06353 & 0,08578 & 0,11157 & 0,16151 & 0,20955 & 0,25399 & 0,29456 & 0,33165 & 0,36588 \\
\hline 0,95 & 0,00000 & 0,03798 & 0,08776 & 0,14137 & 0,16687 & 0,19384 & 0,24295 & 0,28824 & 0,33007 & 0,36898 & 0,40545 & 0,43975 \\
\hline \multicolumn{13}{|l|}{ At-the-money } \\
\hline 1,00 & 0,00000 & 0,05239 & 0,11020 & 0,16712 & 0,18695 & 0,22027 & 0,26899 & 0,31372 & 0,35515 & 0,39387 & 0,43030 & 0,46975 \\
\hline \multicolumn{13}{|l|}{ In-the-money } \\
\hline 1,05 & 0,00000 & 0,13144 & 0,18919 & 0,24351 & 0,26873 & 0,29406 & 0,34121 & 0,38538 & 0,42689 & 0,46594 & 0,50269 & 0,54305 \\
\hline 1,10 & 0,00000 & 0,16059 & 0,21805 & 0,27168 & 0,29035 & 0,32174 & 0,36865 & 0,41277 & 0,45431 & 0,49341 & 0,53018 & 0,57056 \\
\hline 1,15 & 0,00000 & 0,23059 & 0,28758 & 0,34104 & 0,36056 & 0,39123 & 0,43839 & 0,48271 & 0,52436 & 0,56349 & 0,60994 & 0,64159 \\
\hline 1,20 & 0,00000 & 0,26058 & 0,31752 & 0,37096 & 0,38979 & 0,42117 & 0,46837 & 0,51271 & 0,55435 & 0,59349 & 0,63995 & 0,67159 \\
\hline
\end{tabular}

Tabela 2 - Valor da opção segundo o modelo de Brennan e Schwartz

\begin{tabular}{|c|c|c|c|c|c|c|c|c|c|c|c|c|}
\hline \multicolumn{13}{|c|}{ CÁLCULO DO VALOR DA OPÇÃO PELO MODELO DE BRENNAN E SCHWARTZ } \\
\hline \multicolumn{13}{|c|}{$\sigma=3,96 \%, r=4,27 \%$ e $C=1,0 \%$} \\
\hline$s / x$ & $t=0$ & $t=3$ & $t=6$ & $t=9$ & $t=10$ & $t=12$ & $t=15$ & $t=18$ & $t=21$ & $t=24$ & $t=27$ & $t=30$ \\
\hline \multicolumn{13}{|l|}{ Out-of-the-money } \\
\hline 0,80 & 0,00000 & 0,00000 & 0,00000 & 0,01247 & 0,03292 & 0,04142 & 0,08239 & 0,12655 & 0,16569 & 0,19526 & 0,21480 & 0,22654 \\
\hline 0,85 & 0,00000 & 0,00000 & 0,02059 & 0,06008 & 0,08302 & 0,10905 & 0,15650 & 0,19503 & 0,22247 & 0,24061 & 0,25267 & 0,26134 \\
\hline 0,90 & 0,00000 & 0,00000 & 0,02902 & 0,07855 & 0,09807 & 0,13419 & 0,18353 & 0,22052 & 0,24530 & 0,26136 & 0,27252 & 0,28119 \\
\hline 0,95 & 0,00000 & 0,04148 & 0,09845 & 0,15662 & 0,17714 & 0,20571 & 0,24228 & 0,26810 & 0,28676 & 0,30110 & 0,31238 & 0,32076 \\
\hline \multicolumn{13}{|l|}{ At-the-money } \\
\hline 1,00 & 0,00000 & 0,05782 & 0,12503 & 0,18628 & 0,20452 & 0,23384 & 0,26763 & 0,29154 & 0,30966 & 0,32428 & 0,33595 & 0,34401 \\
\hline \multicolumn{13}{|l|}{ In-the-money } \\
\hline 1,05 & 0,00000 & 0,13831 & 0,19929 & 0,24868 & 0,22051 & 0,28619 & 0,31468 & 0,33695 & 0,35441 & 0,36747 & 0,37627 & 0,40664 \\
\hline 1,10 & 0,00000 & 0,16963 & 0,22967 & 0,27669 & 0,29586 & 0,31262 & 0,34079 & 0,36339 & 0,38114 & 0,39410 & 0,40249 & 0,43306 \\
\hline 1,15 & 0,00000 & 0,23493 & 0,28847 & 0,33081 & 0,34919 & 0,36413 & 0,39021 & 0,41006 & 0,42427 & 0,43352 & 0,47759 & 0,48379 \\
\hline 1,20 & 0,00000 & 0,26497 & 0,31795 & 0,36017 & 0,37058 & 0,39369 & 0,41990 & 0,43958 & 0,45341 & 0,46220 & 0,50657 & 0,51609 \\
\hline
\end{tabular}


potencialidade de maior nível de produção, chegamos à conclusão de que o incremento de poços injetores é limitado. A partir de três poços injetores, o Caso0 é o melhor caso, dado o alto custo operacional e de desenvolvimento. Contudo, as técnicas de simulação de reservatório evidenciam que a melhor solução é o CasoGas com um poço injetor. A Figura 4 mostra o quanto essas técnicas de engenharia de reservatórios podem agregar valor para a empresa proprietária do reservatório.

\section{Análise de sensibilidade}

Como toda opção financeira, os valores dos reservatórios precificados segundo a teoria da opção são sensíveis às variações de seus parâmetros ${ }^{14}$, tais como: a) taxa livre de risco; b) custos operacionais e de desenvolvimento; c) tempo de concessão; d) convenience yield e/ou dividend yield; e e) volatilidade do preço (valor) do petróleo.

Como exercício, variamos cada um desses parâmetros em $1 \%$, a fim de determinar a "elasticidade" do valor da opção em relação a cada um deles. Os resultados estão apresentados na Tabela 3.
Notamos que o valor do reservatório é sensível à taxa de convenience e/ou dividend yield, bem como ao tempo até expirar a concessão. Isso evidencia que os termos de concessão são importantes no processo de licitação (nível de royalties e tempo da concessão).

\section{CONSIDERAÇÕES FINAIS}

Este estudo teve como objetivo incluir flexibilidade gerencial (tais como técnicas de injeção de gás e água) na avaliação de reservatórios. Concluímos que essas técnicas podem aumentar o valor dos reservatórios em até $25 \%$ segundo a teoria de opções reais. A principal vantagem da metodologia de teoria de opções em relação à tradicional técnica de fluxo de caixa descontado é levar em conta as questões operacionais da indústria do petróleo.

Utilizamos dois modelos clássicos para a precificação de reservatórios de petróleo e aplicamos uma análise de sensibilidade para determinar quais fatores são mais relevantes no seu valor econômico. Como era de se esperar, em ambos os modelos, o tempo de con-

\section{Figura 4 - Valor do reservatório segundo as três técnicas}

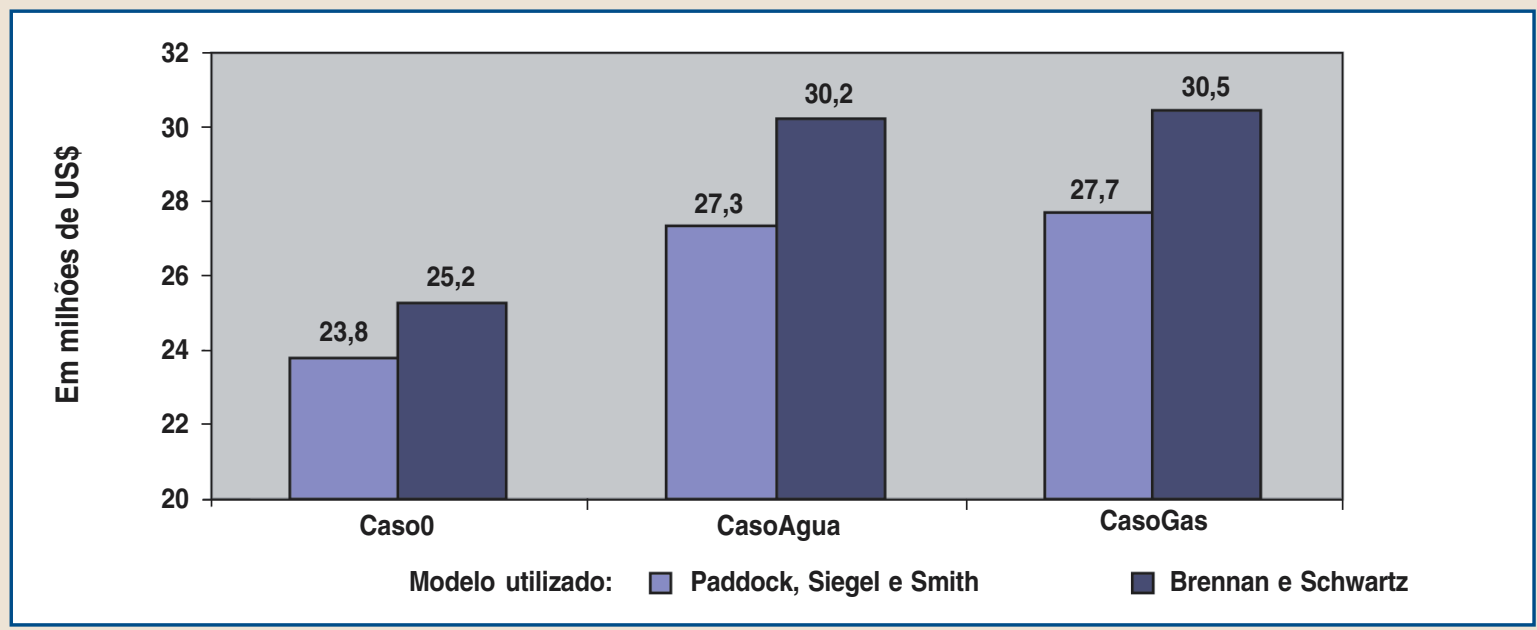

Tabela 3 - Análise de sensibilidade

\begin{tabular}{|l|c|c|}
\hline Parâmetro & $\begin{array}{c}\text { Modelo de Brennan } \\
\text { e Schwartz (\%) }\end{array}$ & $\begin{array}{c}\text { Modelo de Paddock, } \\
\text { Siegel e Smith (\%) }\end{array}$ \\
\hline Taxa livre de risco & 1,25 & 4,32 \\
\hline Convenience yield & $(3,13)$ & - \\
\hline Incremento no dividendo & - & $(1,19)$ \\
\hline Volatilidade do preço do petróleo & 0,11 & 2,52 \\
\hline Tempo até expirar a concessão & 2,88 & $\mathbf{8 , 7 4}$ \\
\hline
\end{tabular}


cessão e a taxa de convenience e/ou dividend yield, foram os fatores mais importantes.

O modelo de Brennan e Schwartz (1985) considera a taxa de extração como constante ao longo do tempo. Essa consideração pode ser válida na etapa inicial de produção do reservatório; no entanto, durante todo o período, isso não ocorre. No modelo proposto por Paddock, Siegel e Smith (1988), a taxa de decaimento da produção de petróleo é considerada como exponencial, o que é válido na prática, caso a produção esteja dimensionada para a utilização máxima da capacidade desde o instante inicial. Como isso raramente ocorre, a modelagem não é perfeita. O modelo ideal seria um híbrido das duas considerações, com uma taxa de produção constante nos primeiros anos e depois um decaimento exponencial ao longo do tempo.

Os custos de produção poderiam ser modelados de modo que uma parcela fosse atrelada ao preço do óleo, como é o caso dos royalties, e uma outra parcela fosse fixa, referente aos custos de extração e operacionais. Já quanto ao decaimento da produção, como comentado antes, poderia ser utilizado um modelo híbrido que considerasse uma taxa de extração constante durante um certo período de tempo e depois esta passaria a ser exponencial, refletindo o que ocorre na prática. A avaliação das técnicas de engenharia de reservatórios com injeção em períodos diferentes do início da produção seria relevante para melhores estimativas.

Conforme discutido, a estimação dos parâmetros é a grande fraqueza dos modelos. Seria interessante se uma melhor análise da volatilidade pudesse ser feita, e a consideração do preço como reversível à média ou reversível à média com jumps de Poisson, em vez de estocástico browniano, pudesse ser analisada. $\bigcirc$

\section{BIBLIOGRAFIA}

BRENNAN, Michael J., SCHWARTZ, Eduardo S. Evaluating natural resource investments. Journal of Business, v. 58 , p. 135-57, Jan. 1985.

CONSTANTINIDES, G. M. Market risk adjustment in project valuation. Journal of Finance, n. 33, p. 603-16, 1978.

COX, J., ROSS, S. The valuation of options for alternative stochastic processes. Journal of Financial Economics, v. 3 , n. 1/2, p. 145-66, 1976.

DIXIT, Avinash K., PINDYCK, Robert S. Investment under uncertainty. Princeton, New Jersey: Princeton University Press, 1994.

DIXIT, Avinash K., PINDYCK, Robert S. The options approach to capital investment. Harvard Business Review, v. 73, n. 1, p. 105-15, May/June 1995.

GIBSON, Rajna, SCHWARTZ, Eduardo S. Stochastic convenience yield and the pricing of oil contingent claims. Journal of Finance, v. 45, n. 3, July 1990 .
GRUY, H. J., GARB, F. A., WOOD, J. W. Determining the value of oil and gas in the ground. World Oil, n. 194, p. 105-08, 1982.

HULL, John C. Options, futures, and other derivatives. 3. ed. New Jersey : Prentice-Hall, 1997.

KARLIK, C. W. Parametric estimating of oil and gas production facilities capital costs worldwide. Society of Petroleum Engineers, n. 22.015, 1991.

LOHRENZ, J., PEDERSON, J. A. Searching for, developing, and producing oil and gas reserves. Society of Petroleum Engineers, n. $13.773,1985$.

LUEHRMAN, Timothy A. Investment opportunities as real options. Harvard Business Review, v. 76, n. 4, p. 56-67, July/ Aug. 1994.

McDONALD, R., SIEGEL, D. Asset market equilibrium, investment and price behavior of an exhaustible resource. Evanston : Northwestern University, May 1983. (Working Paper).
OLIVEIRA, Carlos Alberto P. de. Avaliação e gerência de jazidas de petróleo: uma abordagem pela teoria das opções. Dissertação (Mestrado) - Departamento de Engenharia Industrial, PUC-RJ, 1990.

PADDOCK, James L., SIEGEL, Daniel R., SMITH, James L. Option valuation of claims on real assets: the case of offshore petroleum leases. Quarterly Journal of Economics, v. 103, p. 479-508, Aug. 1988.

SAITO, Richard, ROCHMAN, Ricardo. Análise de métodos numéricos para precificação de opções. São Paulo : EAESP/ FGV, 1999. (Relatório do NPP)

SIEGEL, Daniel R., SMITH, James L., PADDOCK, James L. Valuing offshore oil properties with option pricing models. Midland Corporate Finance Journal, v. 5, p. 22-30, Spring 1987.

STAUFER, T. R. Estimating the full costs of oil and gas production. Society of Petroleum Engineers, n. 30.064, 1995.

TRIGEORGIS, L. Real options: managerial flexibility and strategy in resource allocation. 2. ed. Cambridge, Massachusetts: The MIT Press, 1997.

NOTAS

1. 0 termo "opção" é em geral relacionado com opções financeiras (sobre ativos financeiros), tais como opção sobre Ibovespa. Já opções reais incluem ativos reais como ativosobjetos.

2. Interessante notar que os relatórios dos bancos de investimento - ING Barings e Deutsch Bank Research - utilizam o parâmetro de US\$ 3 por barril.

3. Para mais detalhes, veja Gruy, Garb e Wood (1982).

4. De acordo com os artigos 5 e 23 da Lei no 9.478/97 (“Lei do Petróleo"), a União Federal poderá permitir que empresas estatais ou privadas, constituídas sob as leis brasileiras e com sede e administração no país, realizem atividades de exploração e produção de petróleo e gás natural no país mediante contratos de concessão, precedidos de licitação. Essa lei quebra 0 "monopólio", antes privilégio da Petrobras, e possibilita que empresas privadas atuem em todos os segmentos da cadeia produtiva do petróleo, desde a exploração até a distribuição.

5. Trigeorgis (1997) apresenta em detalhes os tipos de opções reais segundo a classificação sugerida no texto.
6. Ao contrário do que se possa pensar, a injeção de água é mais cara do que a injeção de gás. A inserção da água é feita a grandes profundidades, o que acarreta maiores gastos operacionais com energia e manutenção. No entanto, a injeção de gás exige que sejam colocados compressores extras para que a pressão acima do óleo seja maior do que a de baixo, acarretando custos de desenvolvimento maiores.

7. Basta colocar os parâmetros na equação de Black-Scholes: $C=$ US $\$ 544,22 \exp ^{(-0,05)(20)}(0,8498)-$ US $\$ 600 \exp ^{(-0,08)(20)}(0,6030)$ = US\$ 97,08 milhões.

8. A colocação dessas premissas faz-se necessária, pois 0 modelo foi desenvolvido para, além de avaliar as reservas, encontrar a política de produção ótima, a qual não será tratada neste artigo.

9. Convenience yield representa o custo de oportunidade na indústria de petróleo.

10. A depleção ocorre à medida que óleo e gás são extraídos do reservatório, pois os recursos são extraídos sem a sua reposição natural.
11. Outras técnicas de resolução de equações diferenciais são descritas em Saito e Rochman (1999).

12. Por exemplo, o aumento do custo de desenvolvimento para - CasoAgua foi calculado dividindo-se 0 custo de desenvolvimento de um poço (US\$ 7,5 milhões) pela quantidade de barris produzida (35.561.220 barris) chegando-se ao valor de US\$ 0,21 por barril. 0 custo total de desenvolvimento considerado foi de US\$ 5,75 por barril (US\$ 5,54 + US\$ 0,21). Para o CasoGas, foi acrescido o valor de um compressor (US\$ 2,5 milhões) ao custo do poço, implicando um custo de desenvolvimento de US\$ 10 milhões. Dividindo-se esse valor pela produção ( 36.500 .000 barris), o custo adicional é de US\$ 0,27 . 0 custo de desenvolvimento para esse caso é, portanto, de US\$ 5,81 .

13. Os custos de injeção foram fornecidos pelo Centro de Estudos de Petróleo (Cepetro) da Unicamp.

14. As chamadas "gregas" na teoria de opções. 